\title{
LIGHTWEIGHT CONCRETE IN YEMEN
}

\section{Hassan S. Abdul-Moghni}

Civil Engineering Dept., Faculty of Engineering, Sana'a University,

Sana'a, Yemen

(Received January 19, 2010 Acepted February 5, 2010).

Light-weight concrete (LWC) has been successfully used since the ancient Roman times and it has gained its popularity due to its lower density and superior thermal insulation properties Compared with normal weight concrete (NWC).The main objective of the present study is to provide some information on properties of light-weight concrete using Yemeni light-weight aggregate, this study covers the use of light-weight coarse aggregate, two types of fine aggregates, superplasticizer and silica fume in concrete. The effects on density, absorption, compressive and splitting tensile strengths of lightweight concrete are reported. The resulting concretes were seen to have densities varying from 1420 to $1760 \mathrm{~kg} / \mathrm{m} 3$, with the corresponding compressive strengths varying from 13.4 to 22.7 $\mathrm{MPa}$.The results of absorption, show that the mixes containing superplasticizer and silica fume have lower levels of absorption compared to the mixes without admixtures. Further, the mixes made with lightweight fine aggregate have absorption values greater than that mixes made with normal fine aggregate.

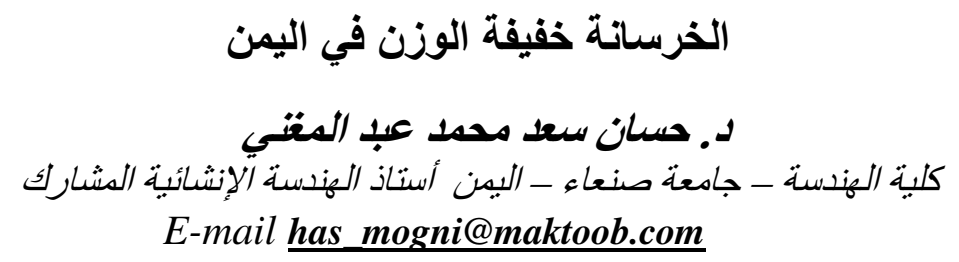

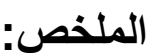

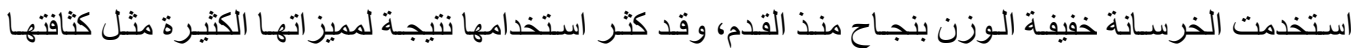

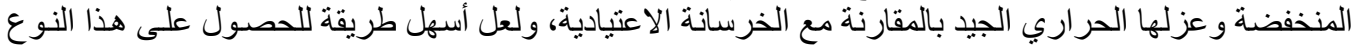

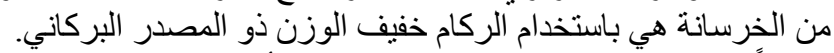

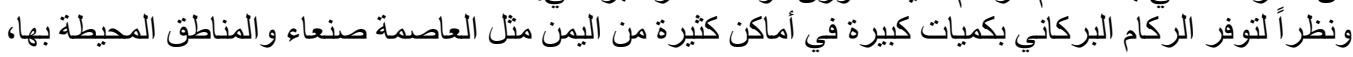

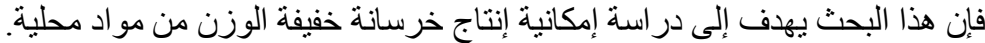

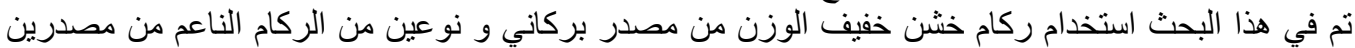

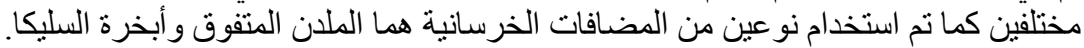

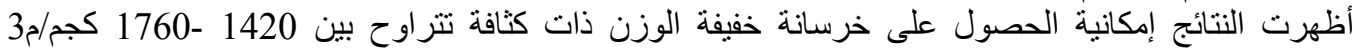

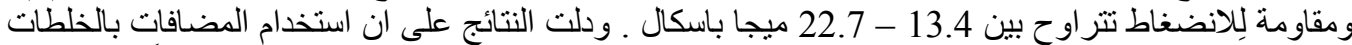

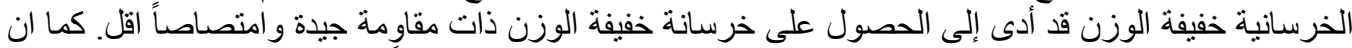

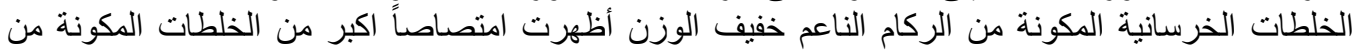

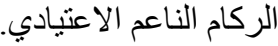

رلكلمـات الدالـة: الركـام الخفيف، الخرسـانة الخفيفة، مقاومـة الخرسـانة ، الكثافـة، الامتصـاص، المضـافات الخرسانية. 
تعرف الخرسانة الخفيفة (LWC) على أنها الخرسانة التي تقل كثافتها عن كثافة الخرسانة العادية حيث تثراوح

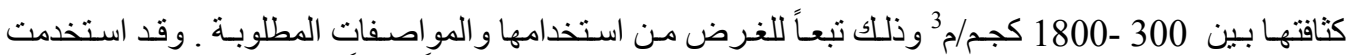

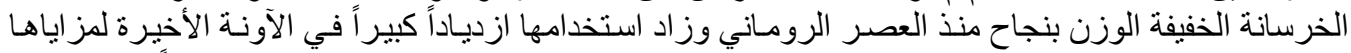

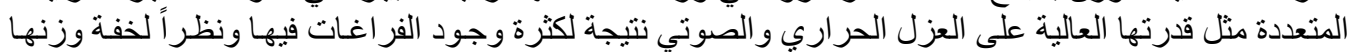

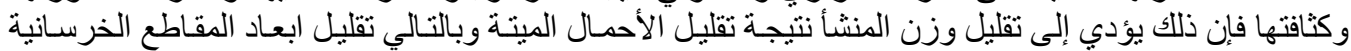

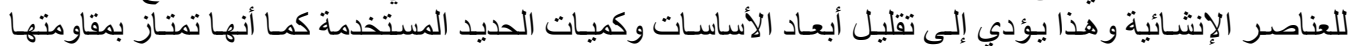

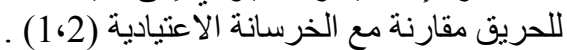

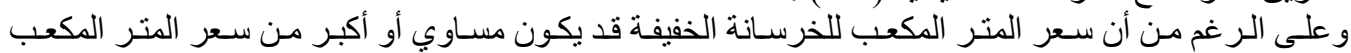

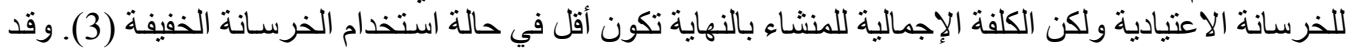

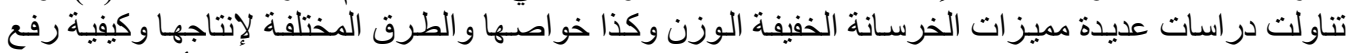

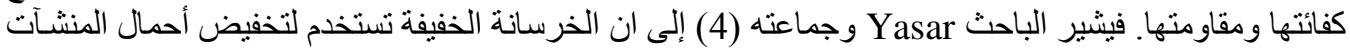

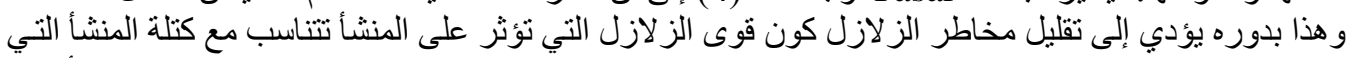

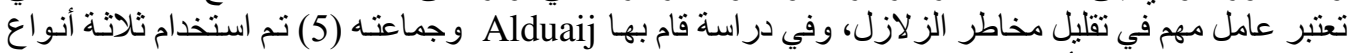

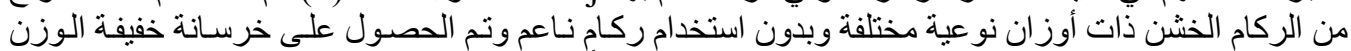

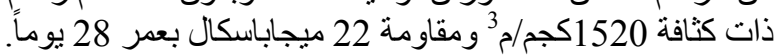

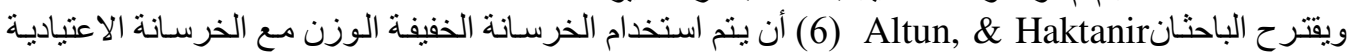

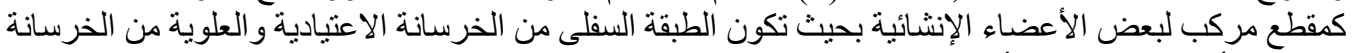

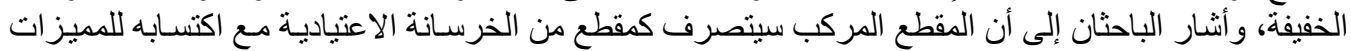

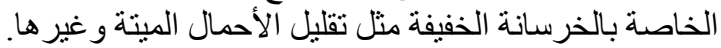

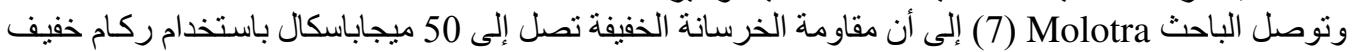

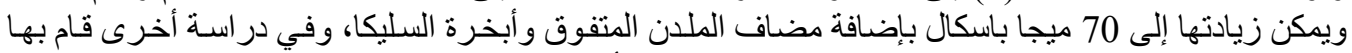

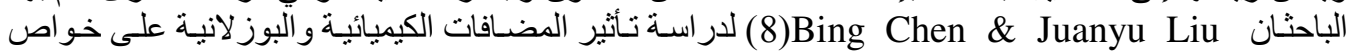

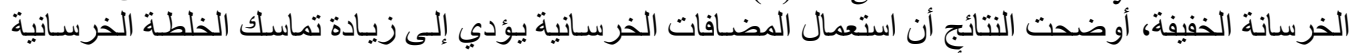
وبالتالي إلى زيادة المقاومة في الأعمار المبكرة و النهائية.

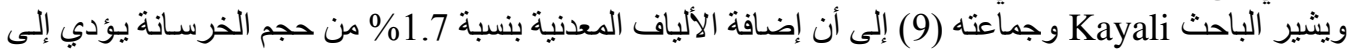

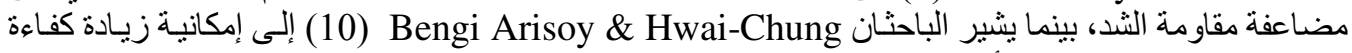

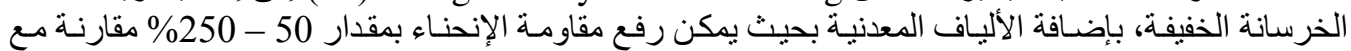

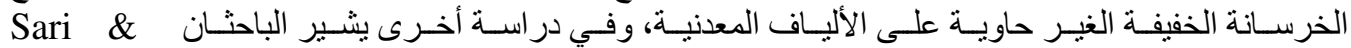
والإلو Pasamehmetoglu

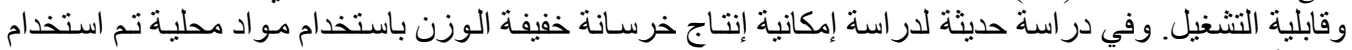

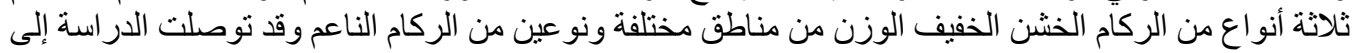

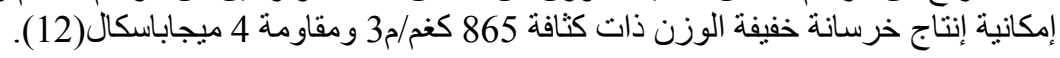

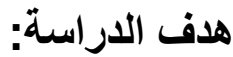

إنتاج خرسانة خفيفة الوزن باستخدام الركام البازلتي الفقاعي خفيف الوزن المتو اجد بكثرة في المناطق اليمنية، كما

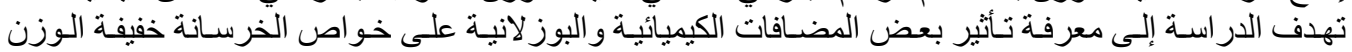

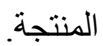

\section{أهمية الدراسة: - ماسة}

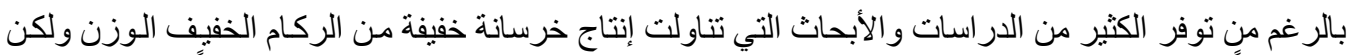

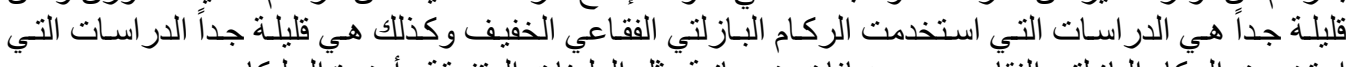
استخذمت الركام البازلتي الفقاعي مع مضافات خرسانية متل الملانات المتفو قة وأبخرة السليكا. 


\section{الأعمال المختبريسـة}

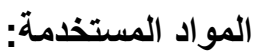

الأسمنت: تم استخدام الأسمنت البورتلاندي الاعتبادي المو افق لمتطلبات المواصفات ASTM C150) لجميع الخلطات الخرسانية. الركام الناعم: تم استعمال نو عين من الركام الناعم:

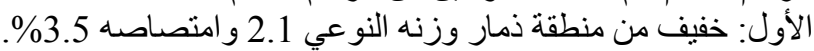

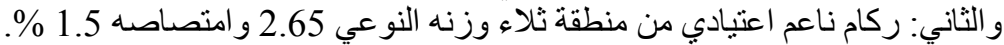

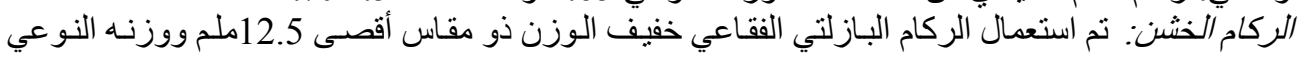

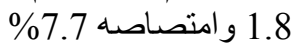
الملان المتفوق: تم استعمال مضاف الملان المتفوق بنسبة 4\% كنسبة من وزن الأسمنت وهو مو افق لمنطلبات

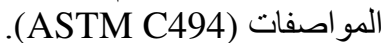
أبخرة السليكا: تم استعمال أبخرة السليكا بنسبة 10\% كنسبة من وزن الأسمنت وهو مطابق لمتطلبات (المواصفات (ASTM C1240-9). تم تحديد نسب $\frac{w}{c}$ لكل الخلطات الخرسانية المستخدمة بحيث تحقق هطول أولي مقداره 60د $20 \pm$ ملم وكانت نسب مكونات الخلطات الخرسانية 1: 1.7: 2 (اسمنت : ركام نـاعم : ركام خشن) كنسب وزنية والجدول (1) يوضح أنو اع الخلطات الخرسانية المستخدمة.

\section{جدول (1) أنواع الخلطات الخرسانية المستخدمة}

\begin{tabular}{|c|c|c|c|c|}
\hline نوع وكمية المضاف المستخدم & الناعكام & $\frac{W}{C}$ & الرمز & اسم الخلطة \\
\hline- & خفيف & 1.23 & M1 & مرجعية مرجية \\
\hline 4\% ملدن متفوق & خفيف & 0.9 & M2 & حاوية على الملدن المتفوق \\
\hline 4\% ملدن+10\% أبخرة السليكا & خفيف & 0.97 & M3 & حاوية على أبخرة السليكا \\
\hline- & اعتيادي & 1.1 & M4 & مرجعية \\
\hline 4\% ملدن متفوق & اعتيادي & 0.77 & M5 & حاوية على الملدن المتفوق \\
\hline 4\% ملدن+10\%\%ٔبخرة السليكا & 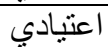 & 0.8 & M6 & حاوية على أبخرة السليكا \\
\hline
\end{tabular}

\section{النتائج والمناقشة}

الكثاف

من خلال النتائج الموضحة بالجدول (2) و الثكل(1) يلاحظ أن الخلطات المنتجة قد حققت المتطلبات الخاصـة

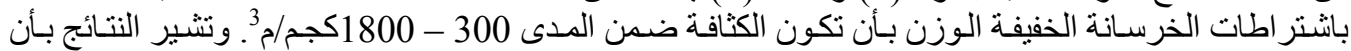

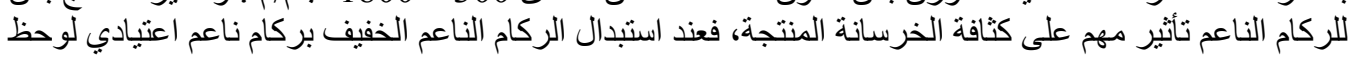

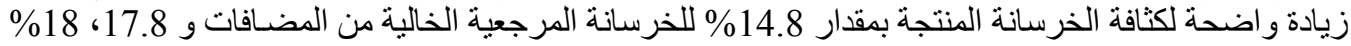

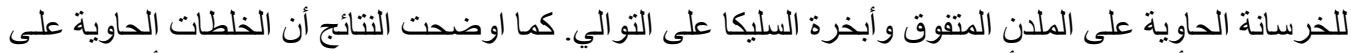

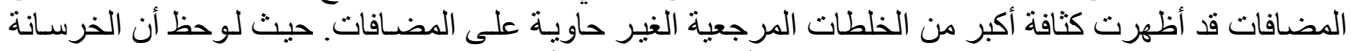

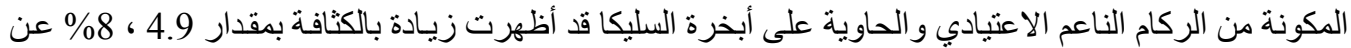

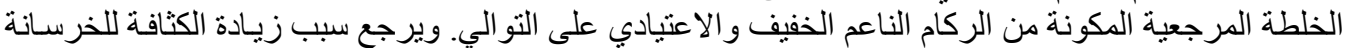

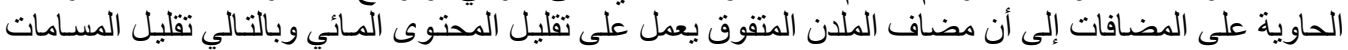




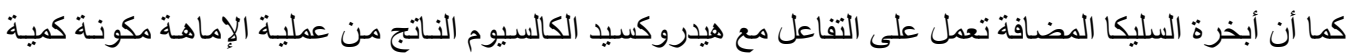
إضافية من الجيل (Gel) التي تعمل بدور ها على سد المسامات وتقلبلها وبالتالي زيادة الكثافة (13).

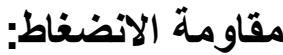

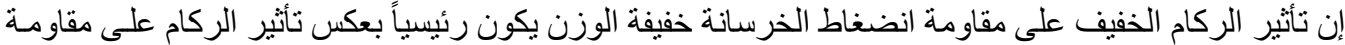

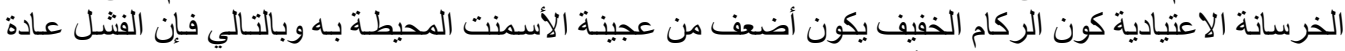

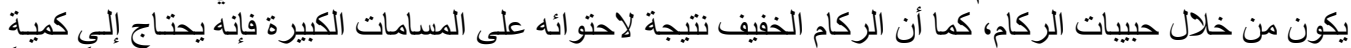

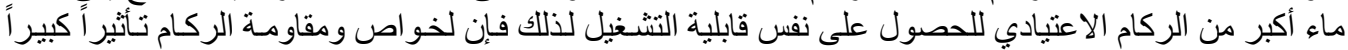

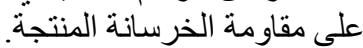

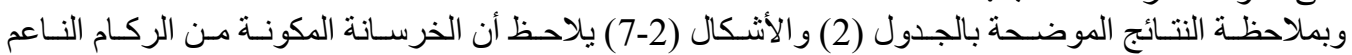

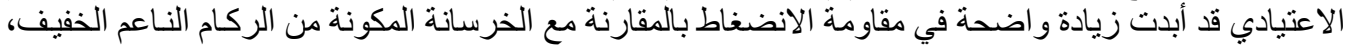

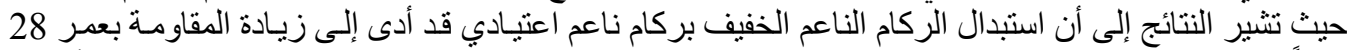

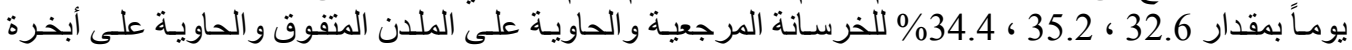

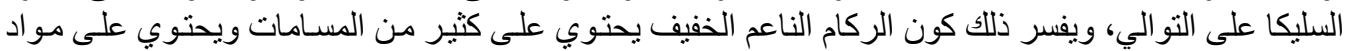
ناعمة بشكل كبير وذلك يؤدي إلى زيادة مـاء الخلط ممـا يؤدي إلى تقليل المقاومـة، لإن المقاومـة ترنبط ارتباط

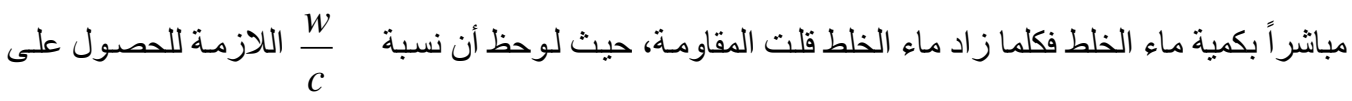
قابلية التشغيل المطلوبـة لخلطة الركام الناعم الخفيف قد تراوحت من 0.97 - 1.23 بينما الخلطة المكونـة من الركام الناعم الاعتيادي تراوحت نسبة $\frac{w}{c}$ من 0.8 - 1.1 للحصول على نفس قابلية التثغيل، و هذا يفسر سبب زيادة الكقاومة للخطات الحاوية على الركام الناعم الاعنيادي.

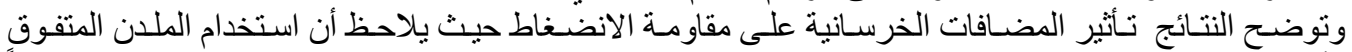

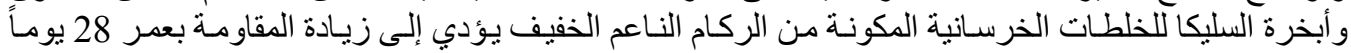

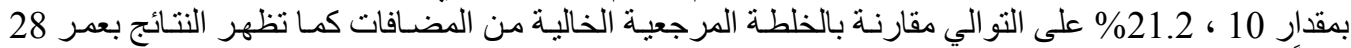

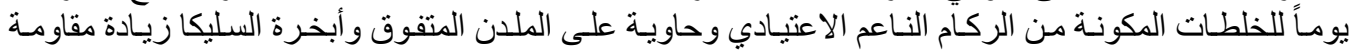

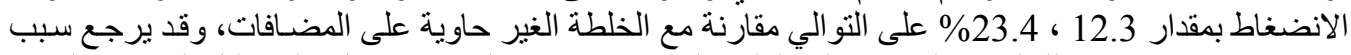

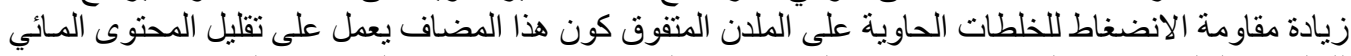

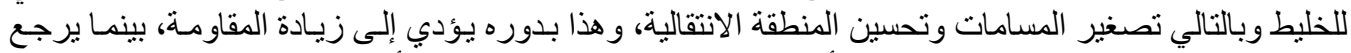

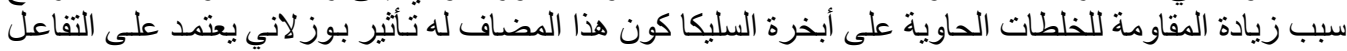

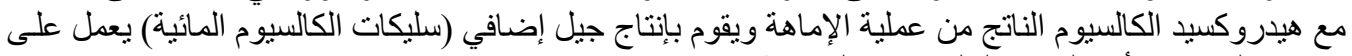
تصغير المسامات أو غلقها وبالنالي زيادة المقاومة.

\section{جدول (2) نتائج مقاومة الانضغاط والكثافة للخلطات الخرسانية}

\begin{tabular}{|c|c|c|c|c|c|}
\hline الكثافة & \multicolumn{4}{|c|}{ مقاومة الانضغاط MPa للعمر (يوم) } & \multirow{2}{*}{ رمز الخلطة } \\
\hline كجم/23 & 90 & 28 & 7 & 1 & \\
\hline 1420 & 13.4 & 12.9 & 11.7 & 3.7 & M1 \\
\hline 1460 & 13.1 & 14.2 & 12.1 & 4 & M2 \\
\hline 1490 & 16.3 & 15.7 & 12.4 & 4.2 & M3 \\
\hline 1630 & 18.0 & 17.1 & 13.3 & 4.6 & M4 \\
\hline 1720 & 20.5 & 19.2 & 14.3 & 5.2 & M5 \\
\hline 1760 & 22.7 & 21.2 & 17.6 & 6.3 & M6 \\
\hline
\end{tabular}




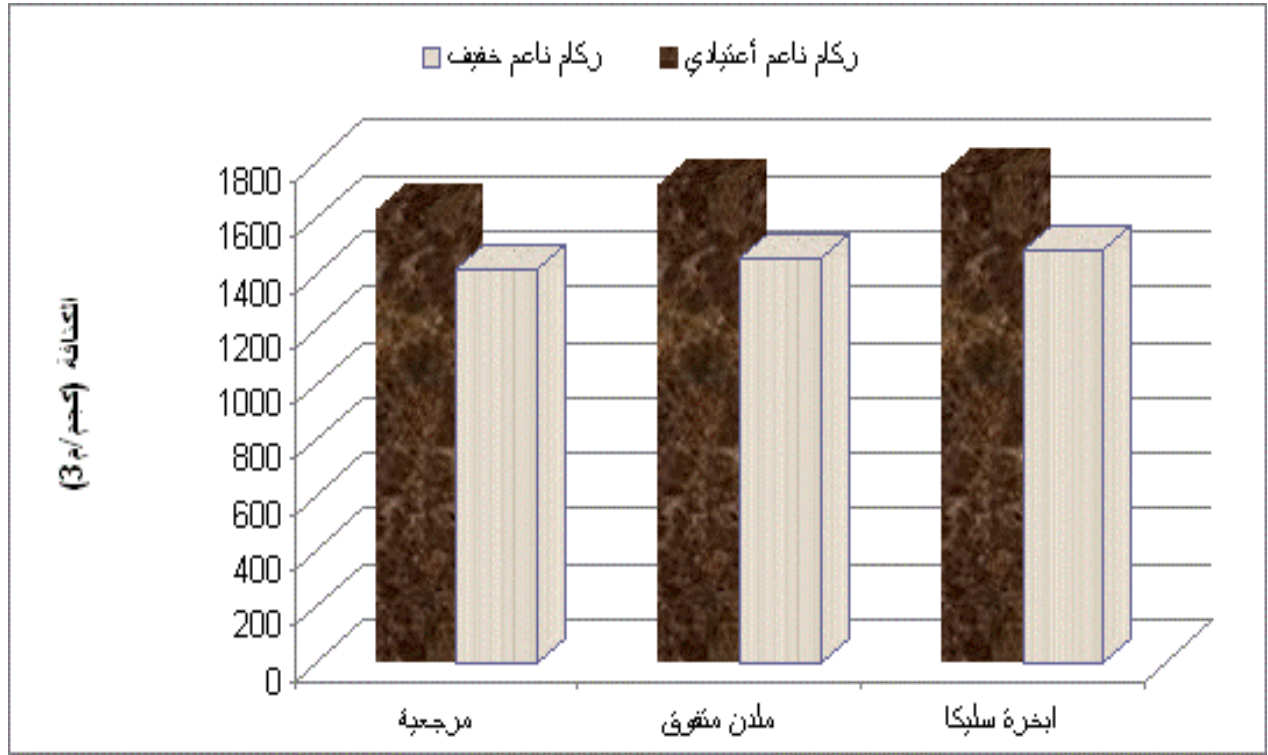

شكل (1) تأثير نوع الركام الناعم على كثافة الخرسانة.

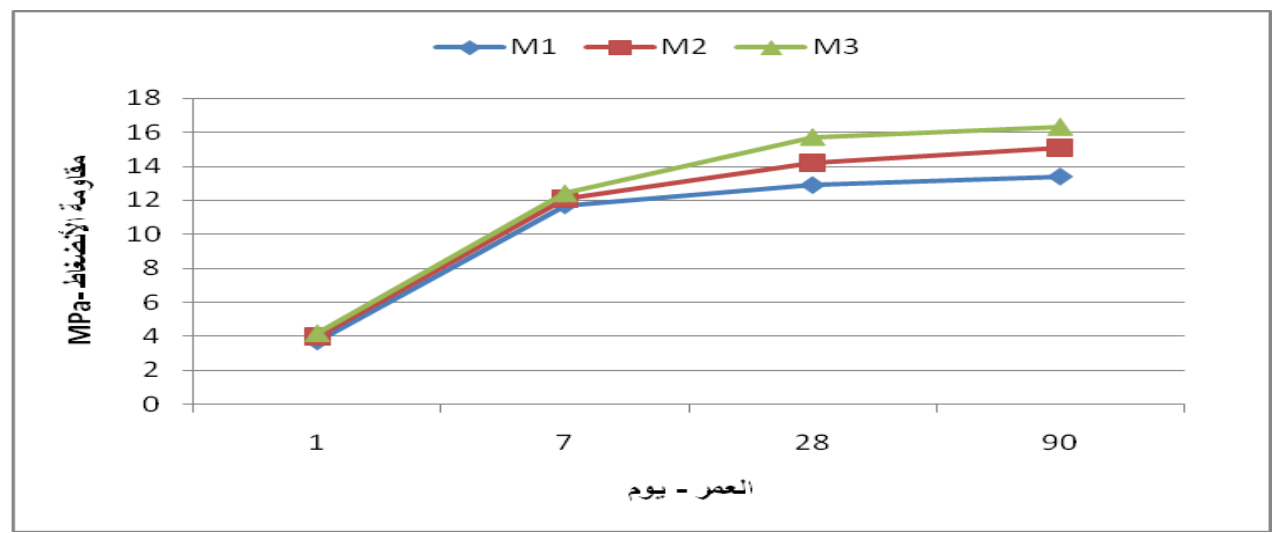

شكل (2) علاقة مقاومة الانضغاط مع العمر للخطات الخرسانية المكونة من الركام الناعم الخفيف

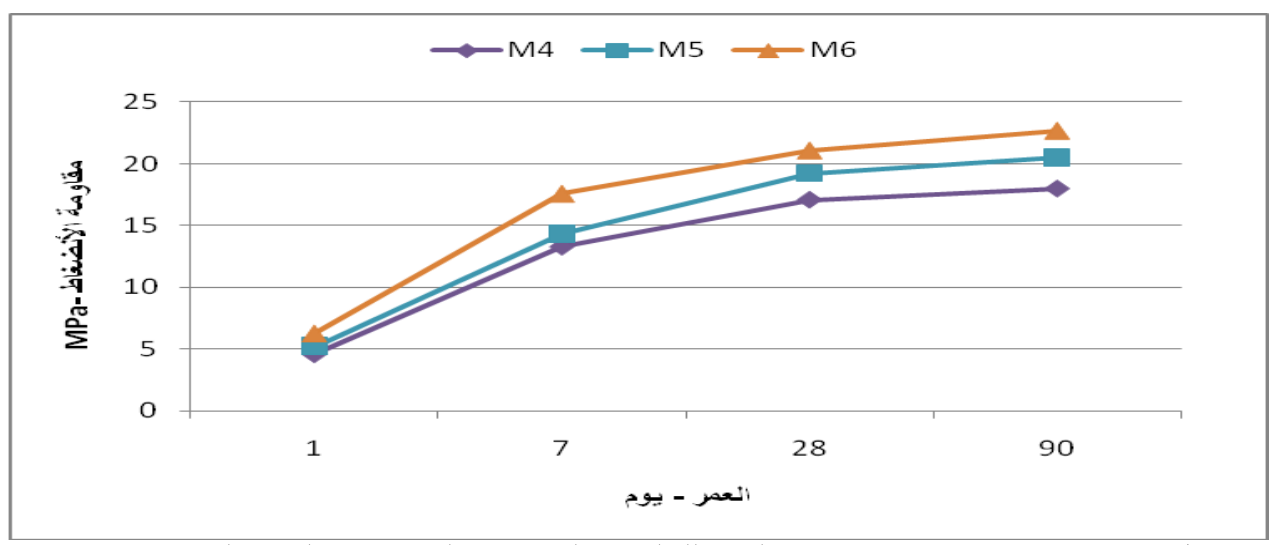

شكل (3) علاقة مقاومة الانضغاط مع العمر للخلطات الخرسانية المكونة من الركام الناعم الاعتيادي لوم 


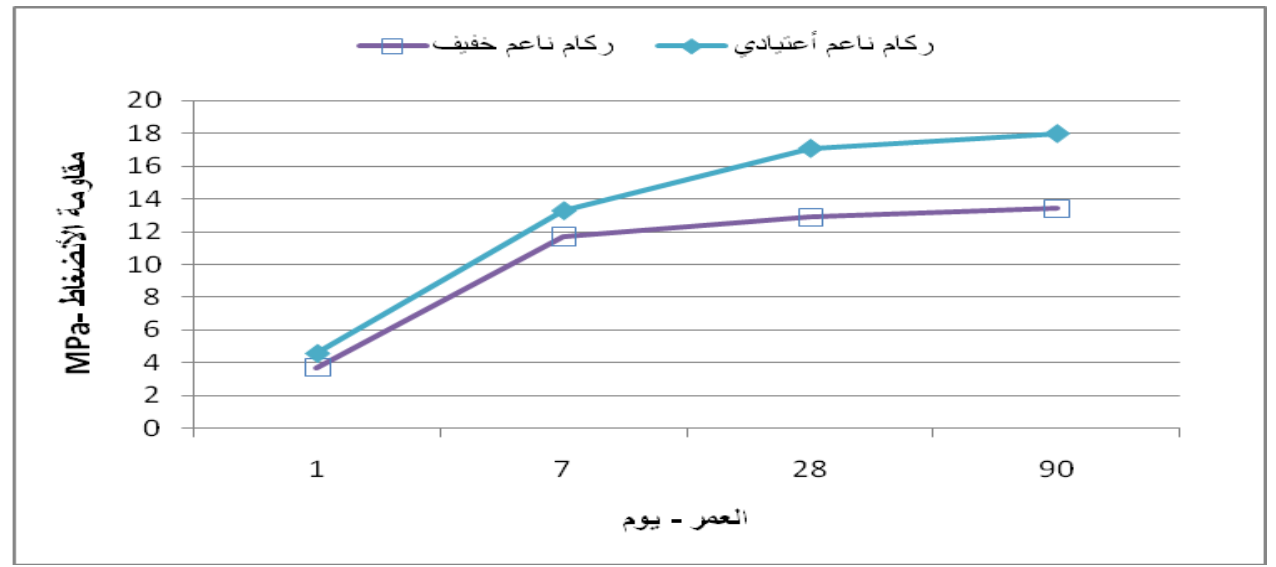

شكل (4) نأثثر نوع الركام الناعم على مقاومة انضغاط الخرسانة المرجعية.

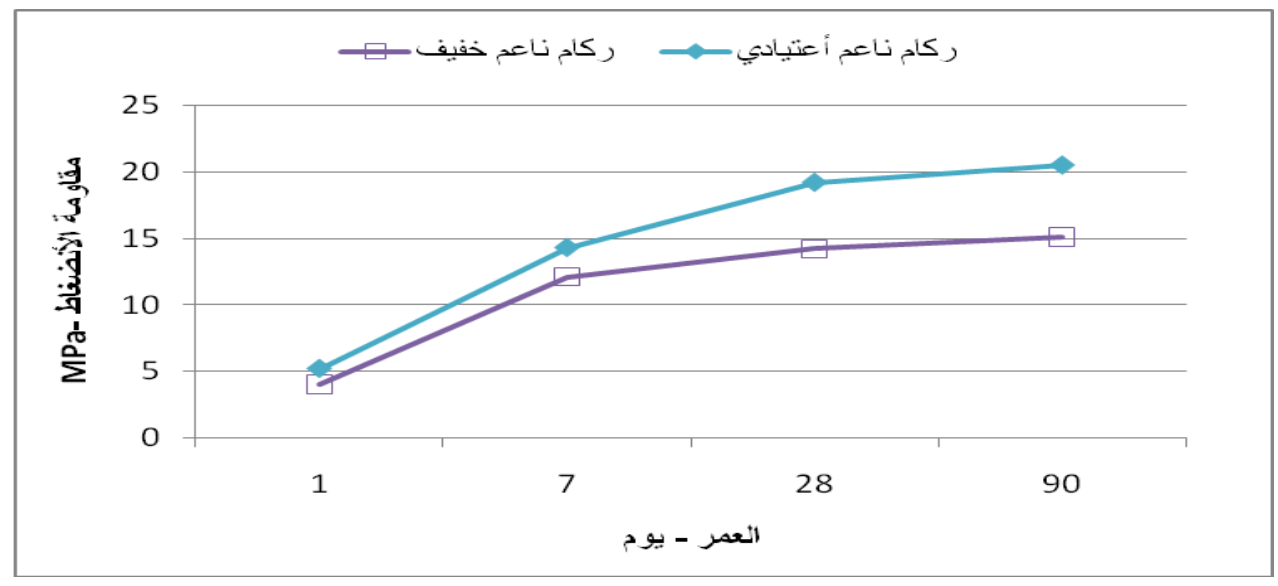

شكل (5) نأثير نوع الركام الناعم على مقاومة انضغاط الخرسانة الحاوية على الملدن المتفوق.

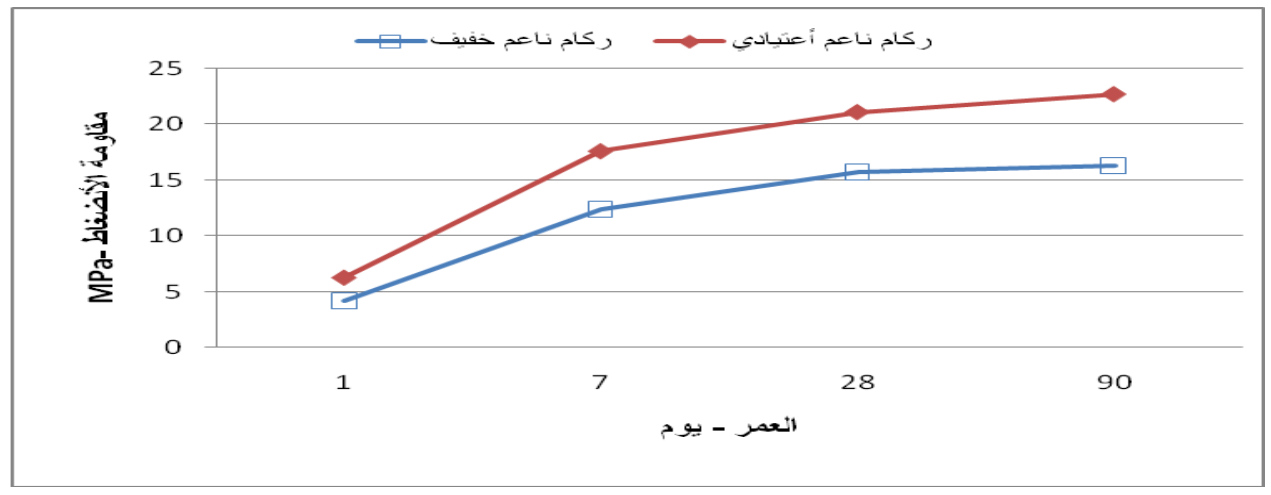

شكل (6) تأثير نوع الركام الناعم على مقاومة انضغاط الخرسانة الحاوية على أبخرة السليكا. 


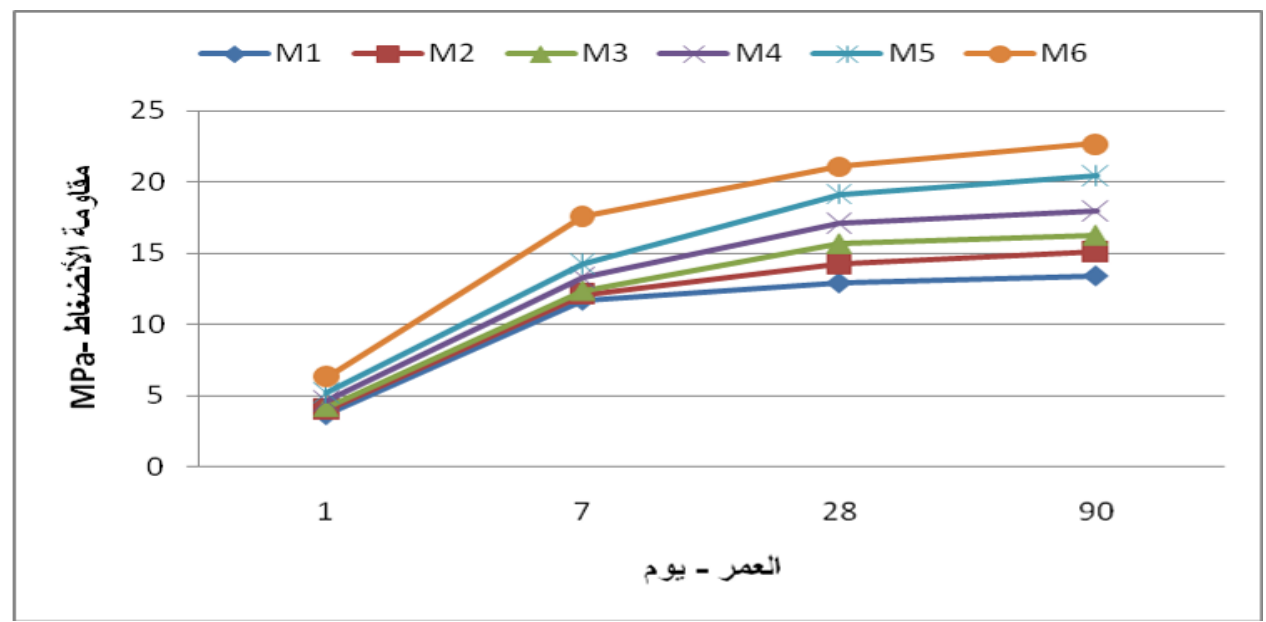

شكل (7) علاقة مقاومة الانضغاط مع العمر للخلطات الخرسانية المختلفة .

مقاومة الثد

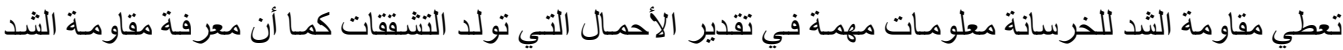

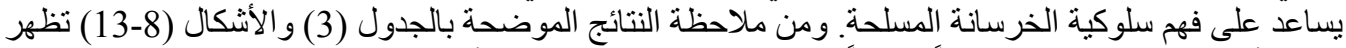

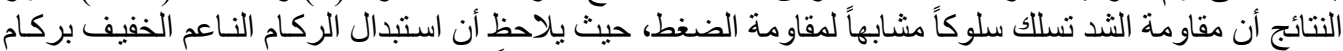

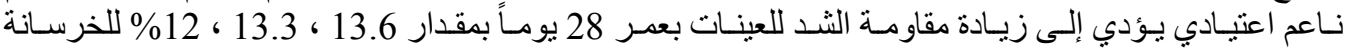

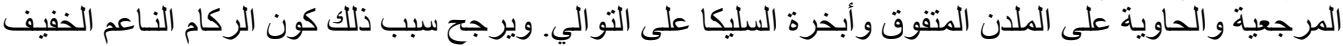

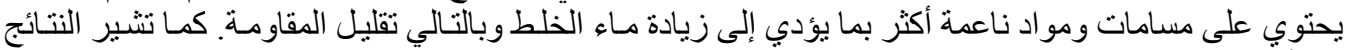

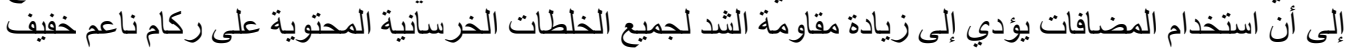

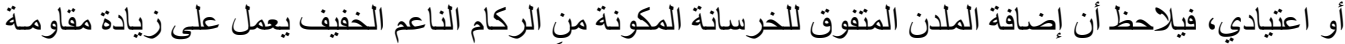

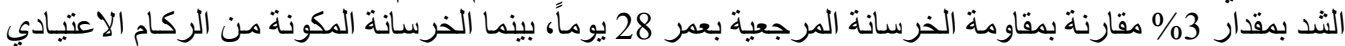

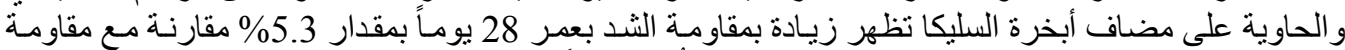

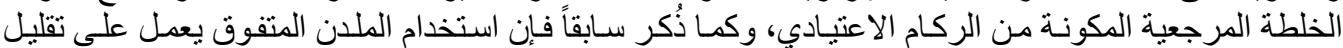

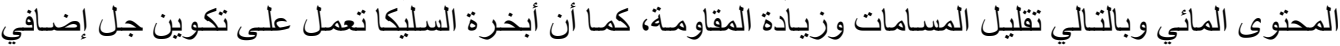

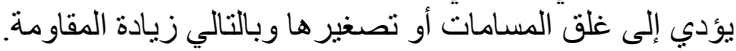

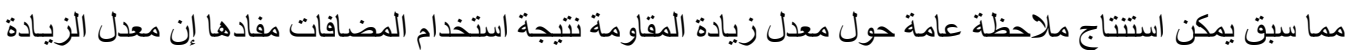

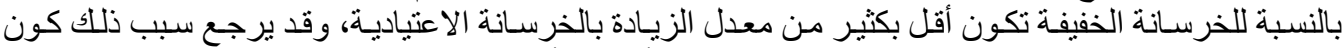

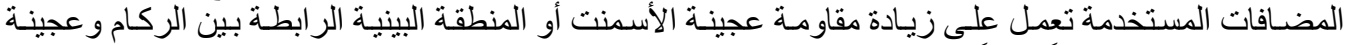

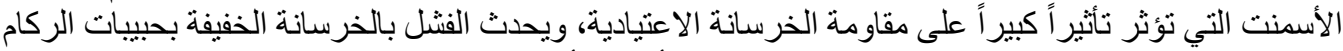

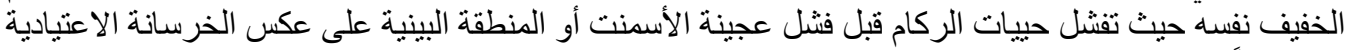
التي غالباً ما يكون الفنشل في المنطقة البينية (14).

\section{| 1امثنصاص}

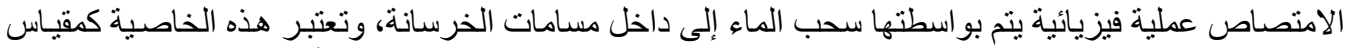

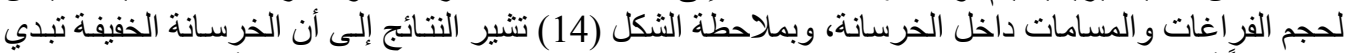

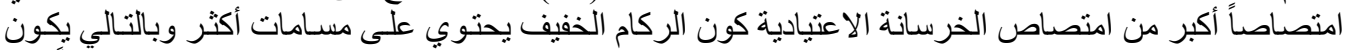

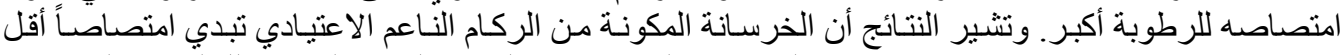

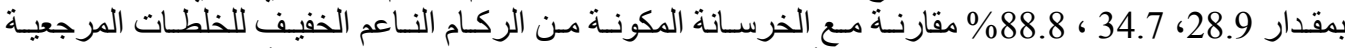

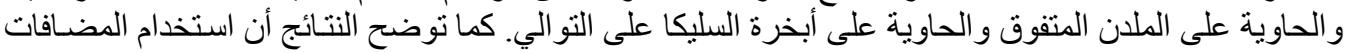


الخرسانية قد عمل على تقليل الامتصاص بصورة واضحة، حيث أن استخدام الملدن المتفوق قد الدئ أدى إلى تقليل

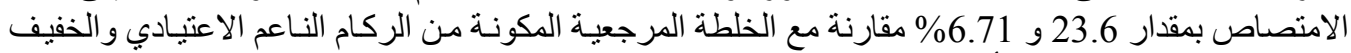

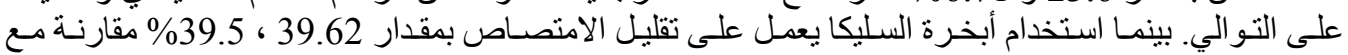

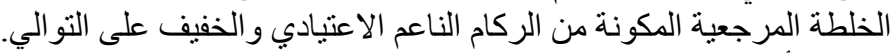

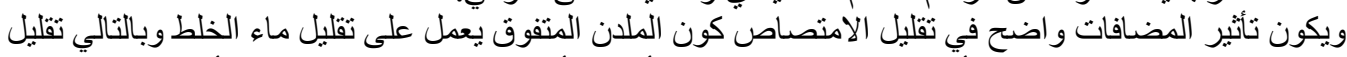

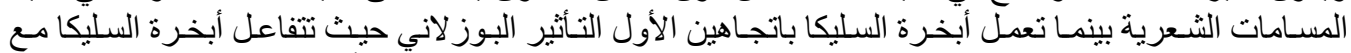

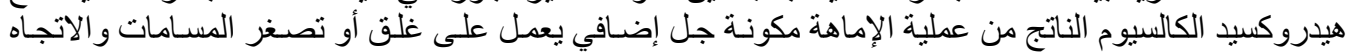

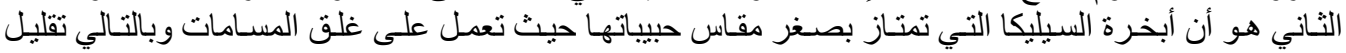

\section{جدول(3) مقاومة الثد والامتصاص للخلطات الخرسانية المستخدمة}

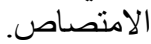

\begin{tabular}{|c|c|c|c|c|}
\hline \multirow{2}{*}{ الامتصاص\% } & \multicolumn{3}{|c|}{ للعمر (يوم)MPaمقاومة الثند } & \multirow{2}{*}{ رمز الخلطة } \\
\hline & 90 & 28 & 7 & \\
\hline 14.9 & 2.42 & 2.34 & 1.98 & M1 \\
\hline 12.41 & 2.48 & 2.41 & 2.26 & M2 \\
\hline 9 & 2.6 & 2.5 & 2.33 & M3 \\
\hline 10.6 & 2.75 & 2.66 & 2.2 & M4 \\
\hline 8.1 & 2.89 & 2.73 & 2.4 & M5 \\
\hline 6.4 & 2.98 & 2.8 & 2.63 & M6 \\
\hline
\end{tabular}

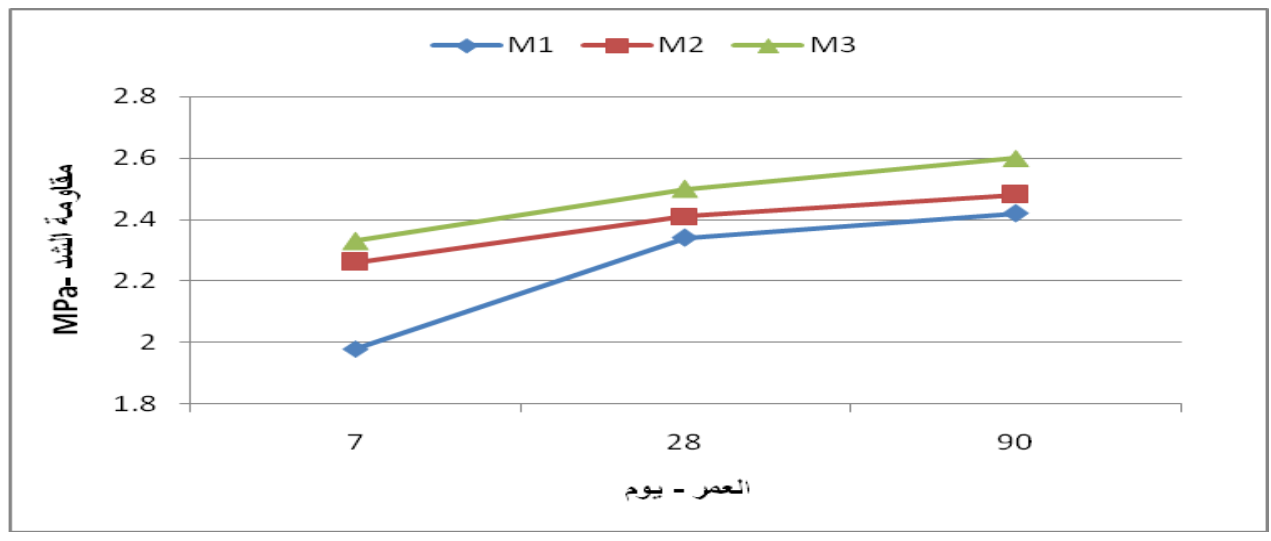

شكل (8) علاقة مقاومة الثند مع العمر للخلطات الخرسانية المكونة من الركام الناعم الخفيف

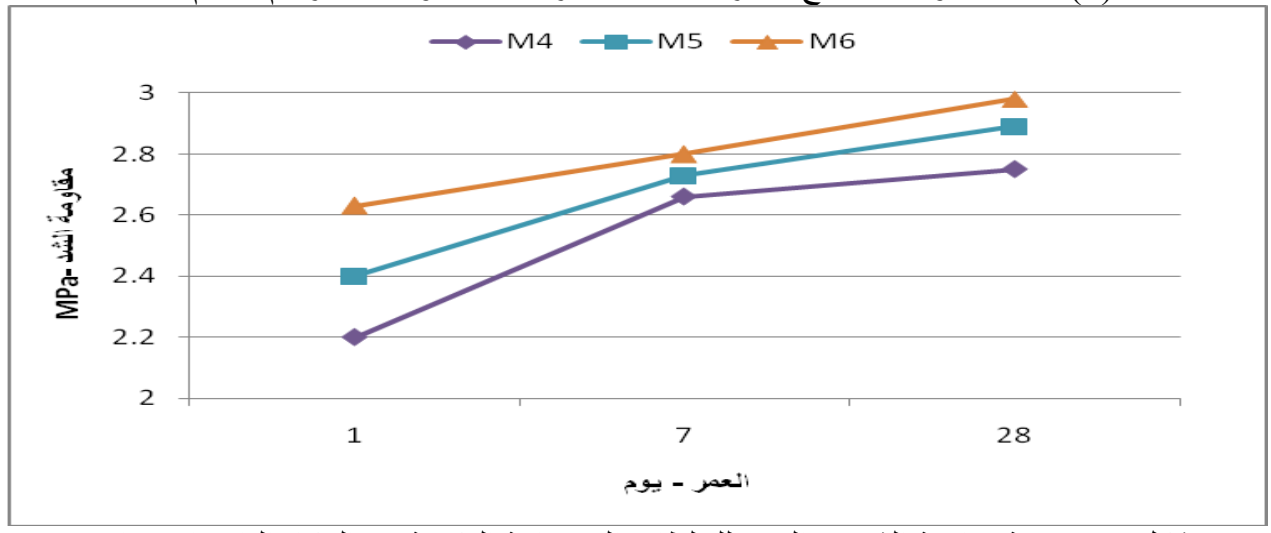

شكل (9) علاقة مقاومة الثد مع العمر للخلطات الخرسانية المكونة من الركام الناعم الاعتيادي 


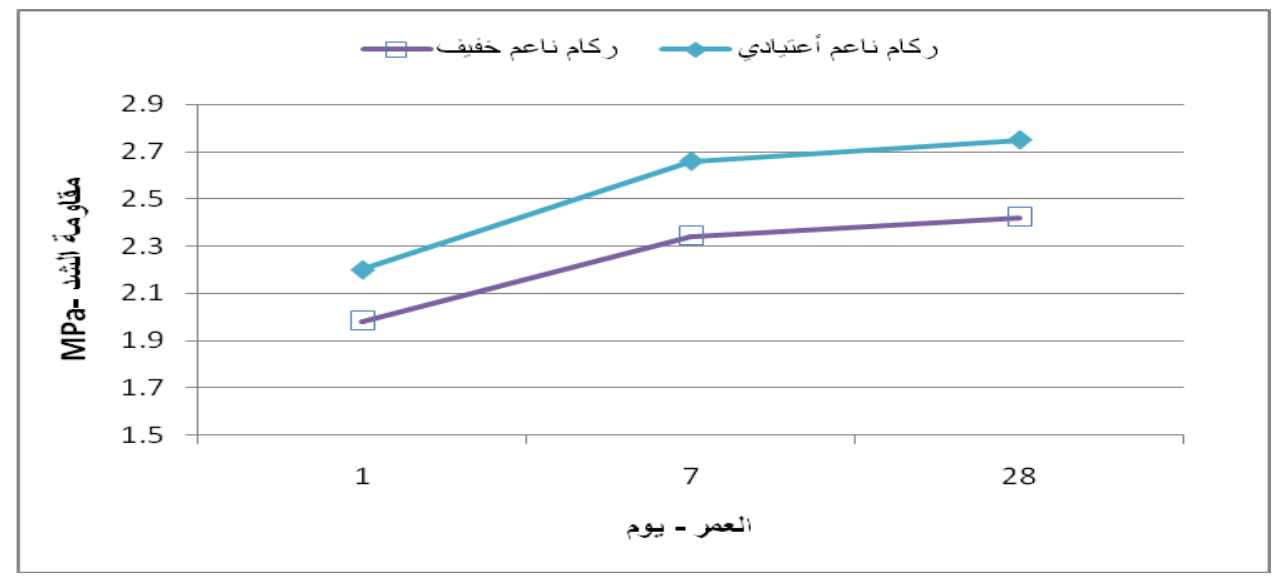

شكل (10) نأثير نوع الركام الناعم على مقاومة الثد للخرسانة المرجعية.

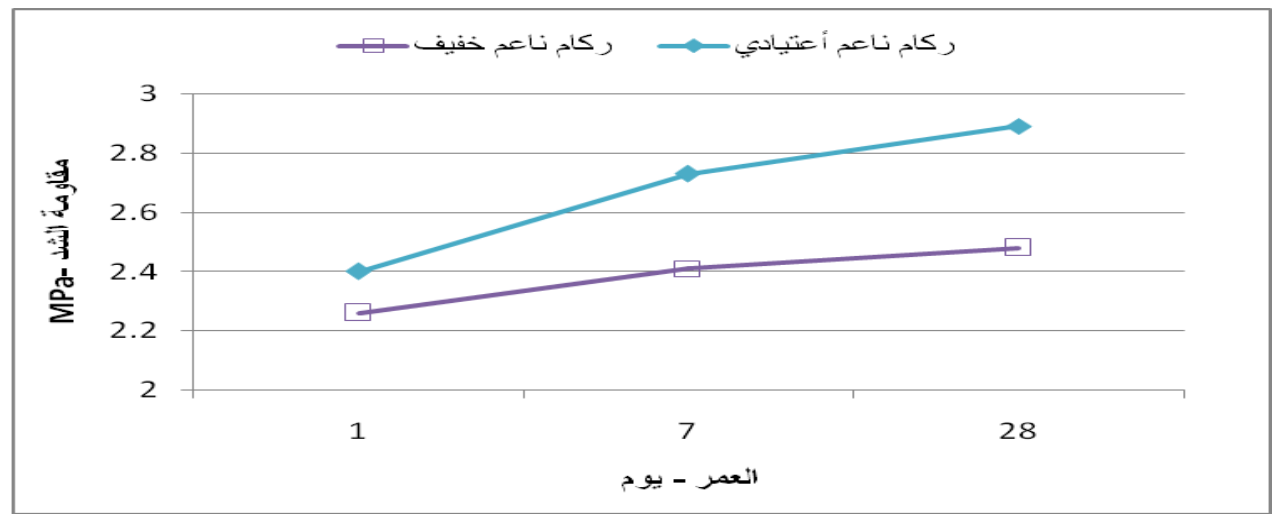

شكل (11) تأثير نوع الركام الناعم على مقاومة الثد للخرسانة الحاوية على الملان المتفوق.

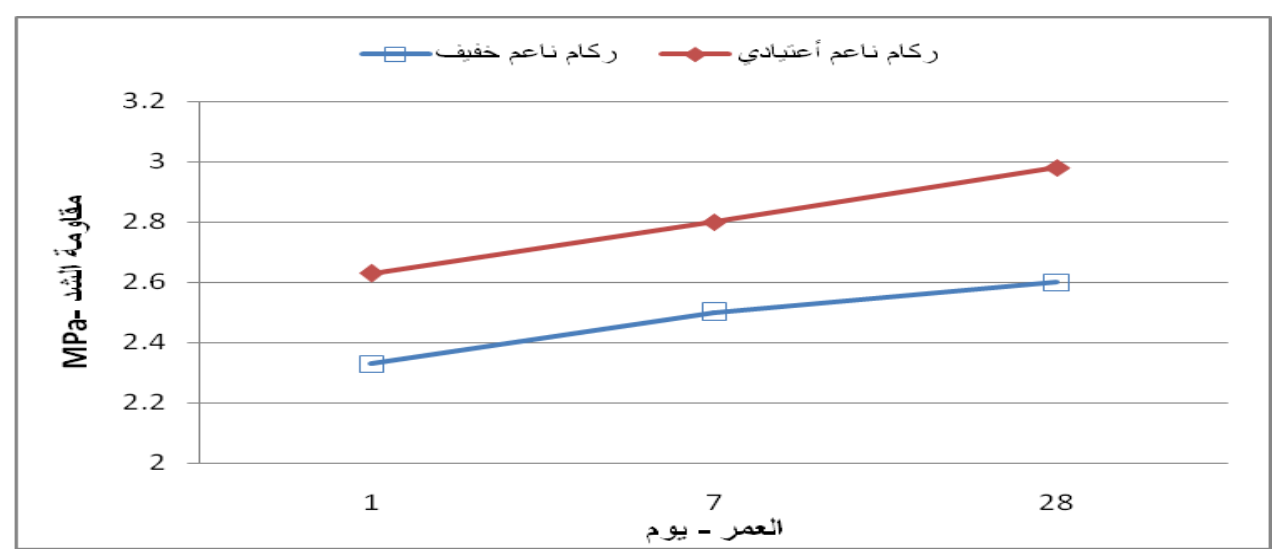

شكل (12) نأثير نوع الركام الناعم على مقاومة الثثد للخرسانة الحاوية على أبخرة السليكا. 


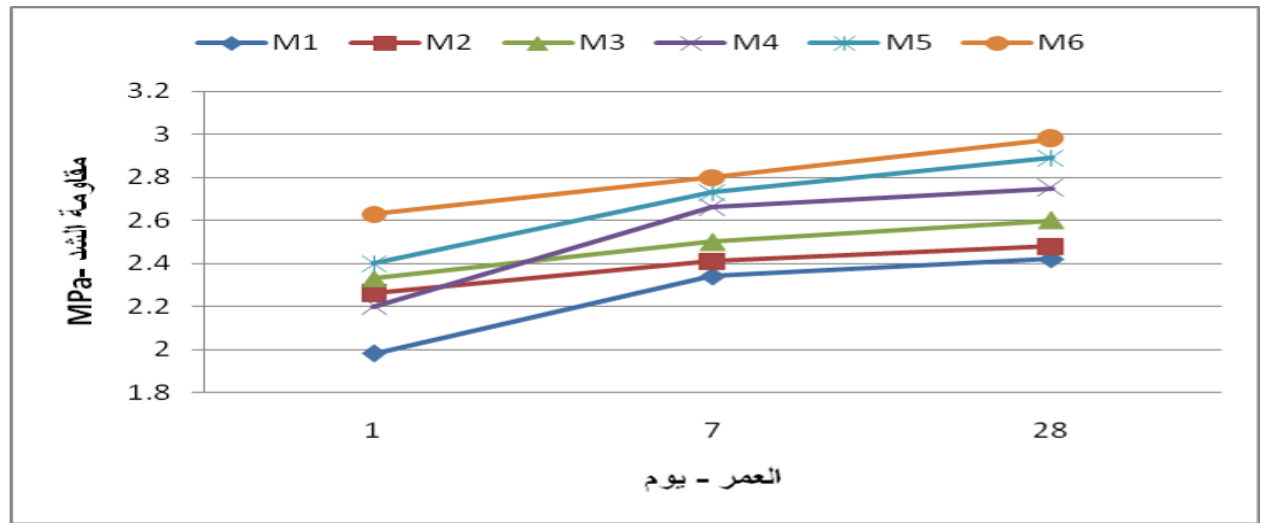

شكل (13) علاقة مقاومة الثد مع العمر للخطات الخرسانية المختلفة .

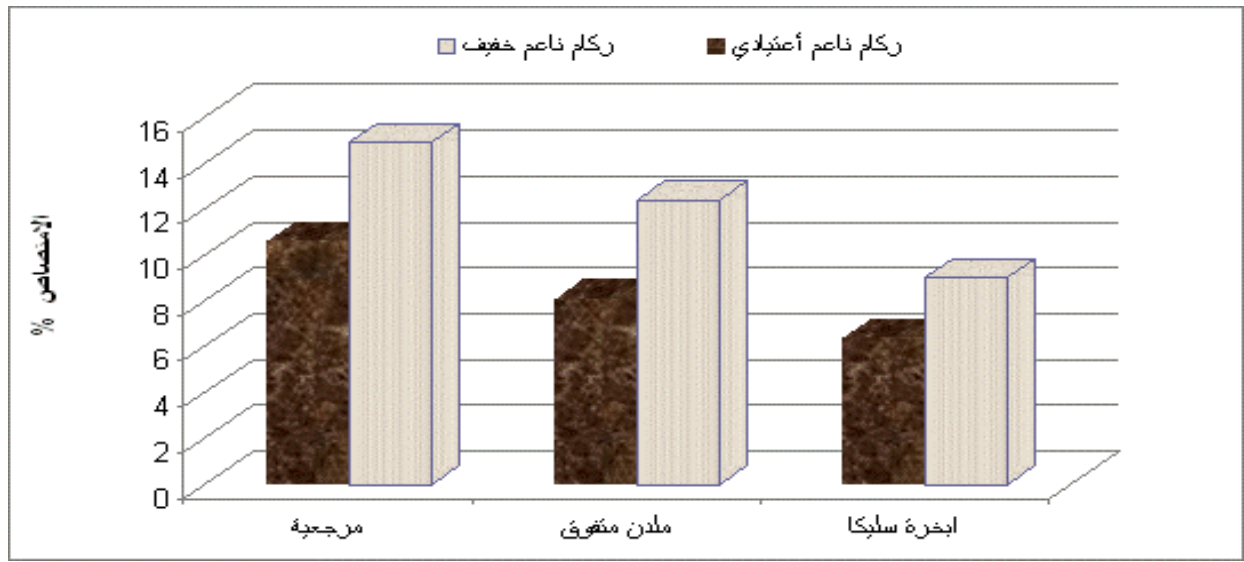

شكل (14) تأثير نوع الركام الناعم على امتصاص الخرسانة.

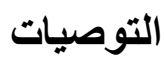

من هذه الدر اسة يمكن استخلاص النتائج التالية:

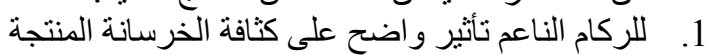

2.

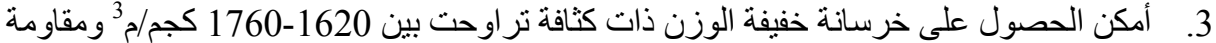

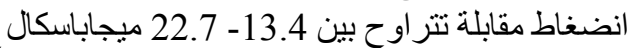

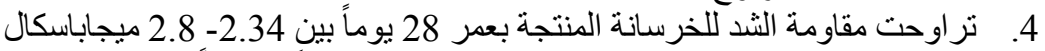

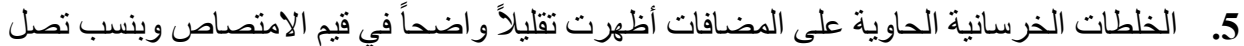

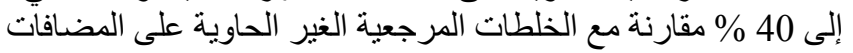

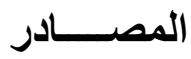

1. Topcu, I.B. "Semi-lightweight concretes produced by volcanic slags" Cem. Concr. Res. 27 (1997) 15- 21.

2. Chandra, S. and Berntsson, L. "Lightweight aggregate concrete" science, technology and applications. Noyes Publications. 
3. Joao A. Rossignolo, Marcos V.C. Agnesini b, Jerusa A. Morais " Properties of highperformance LWAC for precast structures with Brazilian lightweight aggregates" Cement \& Concrete Composites 25 (2003) 77-82.

4. Yasar E, Atis CD, Kilic A, Gulsen "H. Strength properties of Lightweight concrete made with basaltic pumice and fly ash" Materials Letters 2003;57:2267-70.

5. Alduaij, J. Alshaleh, K. Haque, M.N. Ellaithy, K. "Lightweight concrete in hot coastal areas", Cem. Concr. Compos. 21 (1999) 453- 458.

6. Altun, F. Haktanir, T. "Flexural behavior of composite reinforced concrete elements" J. Mater. Civ. Eng. ASCE 13(2001) 255-259.

7. Malhotra, V.M., "CANMET Investigation in the Development of High Strength Concrete Proceedings Utilization of High Strength Concrete," Symposium, Stavanger, Norway, June 1987, pp. 15-86.

8. Bing Chen a, Juanyu Liu "Experimental application of mineral admixtures in lightweight concrete with high strength and workability" Construction and Building Materials 22 (2008) 1108-1113

9. Kayali, O. Haque, M.N. Zhu B. "Some characteristics of high strength fiber reinforced lightweight aggregate concrete" Cement \& Concrete Composites 25 (2003) 207-213

10.Bengi Arisoy a, Hwai-Chung Wu "Material characteristics of high performance lightweight concrete reinforced with PVA" Construction and Building Materials 22 (2008) 635-645

11. Sari, D. Pasamehmetoglu, A.G. "The effects of gradation and admixture on the pumice lightweight aggregate concrete" Cement and Concrete Research 35 (2005) 936-942.

12. Al-Nazily, Ali, "The ability of producing light-weight concrete utilizing indigenous materials" A thesis submitted in partial fulfillment of the requirements for the degree of master of philosophy, Sana'a university, 2009.

13. Alhalf, M.N., and Yousif, H. A., "Concrete Admixtures", University of Technology, Baghdad, 1982.

14.Tommy Y. Lo, W.C. Tang, H.Z. Cui "The effects of aggregate properties on lightweight concrete "Building and Environment 42 (2007) 3025-3029.

15.Tommy Y. Lo, H.Z. Cui, W.C. Tang, W.M. Leung "The effect of aggregate absorption on pore area at interfacial zone of lightweight concrete" Construction and Building Materials 22 (2008) 623-628. 